\title{
Sensibilidad de bacterias procedentes de pasifloras a antibióticos y productos cúpricos
}

\section{Purple passion fruit bacteria sensitivity to antibiotics and copper products}

LINA M. FARFÁN ${ }^{1}$

SOLANGE V. BENÍTEZ2

LILLIANA M. HOYOS-CARVAJAL 3,4

Síntoma de bacteriosis en una hoja de gulupa.

Foto: S.V. Benítez

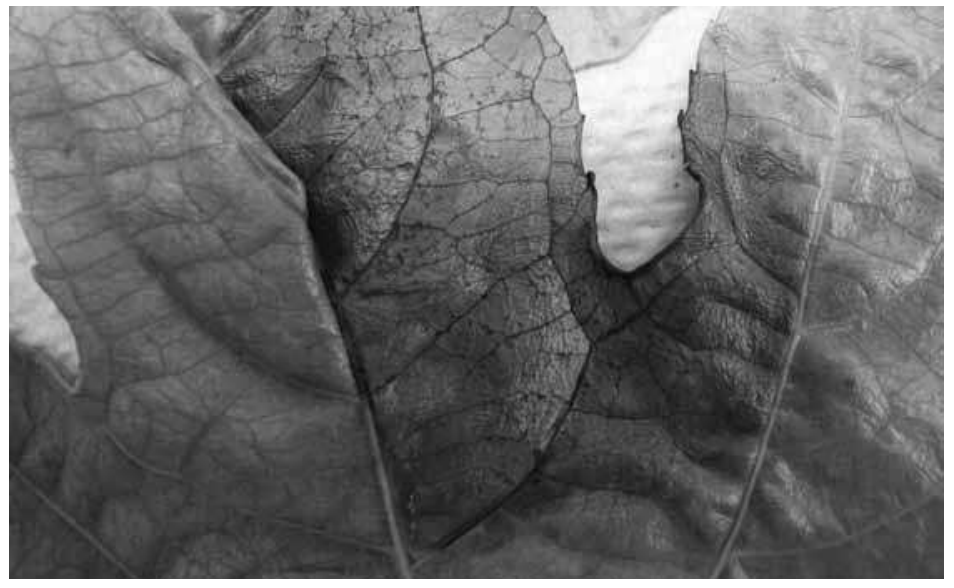

\section{RESUMEN}

La gulupa (Passiflora edulis Sims) es una importante fruta en cuanto a exportaciones en Colombia, esta es atacada por bacterias, entre ellas Xanthomonas axonopodis, pero las alternativas de control basadas en productos cúpricos y antibióticos no han sido eficientes, suponiendo adquisición de resistencia a estos productos por parte de dichos microorganismos. A partir de 111 aislamientos bacterianos asociados a bacteriosis en gulupa y maracuyá (Passiflora edulis var. flavicarpa Deg.) obtenidos en nueve regiones productoras de Colombia, se evaluó por medio de ensayos in vitro la sensibilidad a ampicilina, cloranfenicol, kasugamicina, oxitetraciclina, sulfato de estreptomicina, sulfato de kanamicina y validamicina; y a dos bactericidas cúpricos, oxicloruro y sulfato de cobre. Los resultados indicaron que los mayores porcentajes de sensibilidad a antibióticos, excluyendo el cloranfenicol que no es de uso agrícola, fueron obtenidos con sulfato de estreptomicina $\left(25 \mathrm{mg} \mathrm{L}^{-1}\right)$ con un $79 \%$ de aislamientos sensibles. Toda la población evaluada fue sensible a sulfato de cobre en una concentración de $750 \mathrm{mg} \mathrm{L}^{-1}$; y por el contrario, con oxicloruro de cobre (100\% de la población) presentó resistencia. En general, aproximadamente el $47,9 \%$ de los aislamientos presentó resistencia a los productos evaluados, lo cual correspondería al uso de otras alternativas de control de enfermedades fitobacterianas en el cultivo.

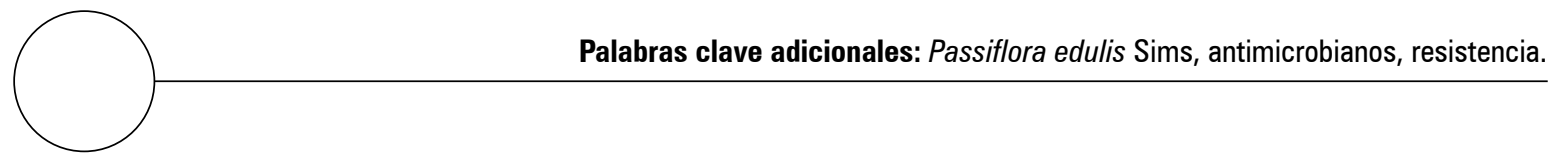

1 Centre for Crop and Disease Management, Department of Environment and Agriculture, Curtin University, Perth (Western Australia).

2 Facultad Ciencias de la Salud, Colegio Mayor de Cundinamarca, Bogotá (Colombia).

3 Facultad de Ciencias Agrarias, Departamento de Ciencias Agronómicas, Universidad Nacional de Colombia, Medellín (Colombia).

$4 \quad$ Autor para correspondencia.limhoyosca@unal.edu.co 


\section{ABSTRACT}

The purple passion fruit (Passiflora edulis Sims.) is an important export fruit in Colombia. It is attacked by bacteria, including Xanthomonas axonopodis, and control alternatives based on copper products and antibiotics have not been effective, assuming acquisition of resistance to these products by these microorganisms. From 111 bacterial isolates associated with bacterial blight in purple passion fruit (Passiflora edulis Sims) and passion fruit (Passiflora edulis var. flavicarpa Deg.) from nine regions of Colombia, an In vitro test was applied to evaluate the sensitivity of strains to ampicillin, chloramphenicol, kasugamycin, oxytetracycline, streptomycin sulphate, kanamycin sulphate and validamycin, and two copper bactericides: oxichloride and copper sulphate. The results showed that the highest rates of sensitivity to antibiotics were obtained with chloramphenicol (50 $\mathrm{mg} \mathrm{L}^{-1}$ ) and streptomycin sulphate ( $25 \mathrm{mg} \mathrm{L}^{-1}$ ), with $83 \%$ and $79 \%$ of susceptible isolates, respectively. Throughout the study the population was sensitive to copper sulphate at a concentration of 750 $\mathrm{mg} \mathrm{L}^{-1}$; and conversely, copper oxychloride (100\% of the population) exhibited resistance. Overall, about $47.9 \%$ of the isolates were resistant to the tested products, which would correspond to the use of alternative controls of phytobacterial disease in this crop.

Additional key words: Passiflora edulis Sims, antimicrobial, resistance.

Fecha de recepción: 28-03-2014

Aprobado para publicación: 03-06-2014
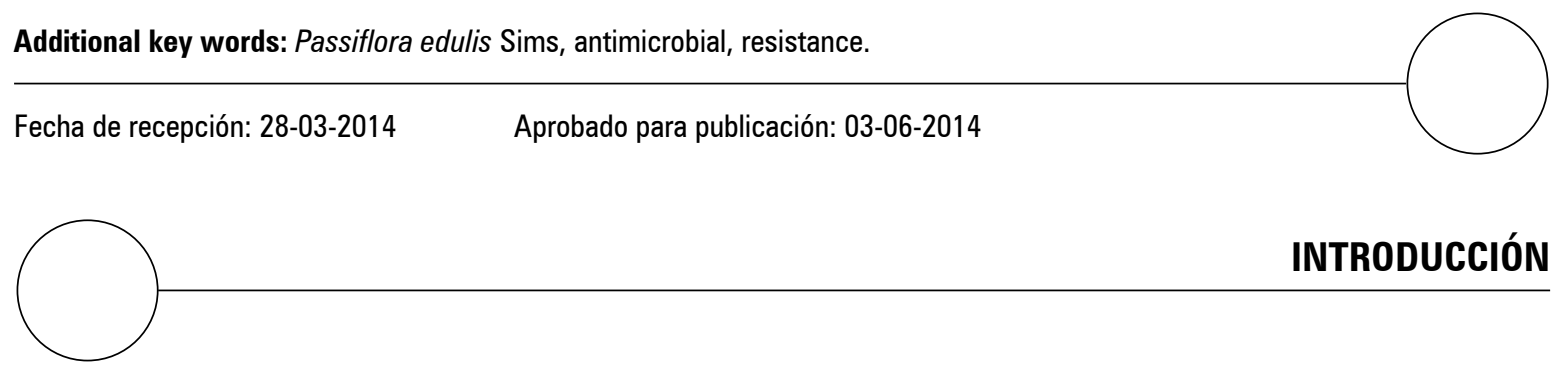

La mancha de aceite, causada por un complejo de bacterias, entre las cuales se encuentra Xanthomonas axonopodis, en Colombia es una de las enfermedades más limitantes en plantaciones de gulupa (Passiflora edulis Sims.) y maracuyá (Passiflora edulis var. flavicarpa Deg.) (Guerrero y Hoyos-Carvajal, 2011). Las pérdidas causadas por esta enfermedad generan reducción de ganancias, erradicación de cultivos y aumento de costos por los elevados precios de los productos químicos empleados como estrategia de control. Adicionalmente, el difícil manejo de esta enfermedad está relacionado con la capacidad del agente causal de infectar varias especies de pasifloráceas (Manicom et al., 2003). Para su control, en Colombia, se realizan aplicaciones no autorizadas de antibióticos como estreptomicina, kasugamicina y validamicina, ya sean solos o en mezcla con productos cúpricos como sulfato y oxicloruro de cobre. En tanto que las evidencias en plantaciones de maracuyá demuestran que la eficacia de estos productos es relativa (Manicom et al., 2003), lo que ha llevado a aplicaciones ge- neralizadas, aumentando el riesgo de generar resistencia en las poblaciones del patógeno a estos productos. La aparición de aislamientos resistentes a agentes de control, ha sido reportada como la principal causa de la reducida eficacia de estos (Llacer et al. 2000; Quezado et al. 2003; Duffy et al., 2011; McGhee y Sundin, 2011.)

Los primeros casos de aislamientos de Xanthomonas sp. resistentes a estreptomicina, fueron reportados en Florida, EE.UU., a principios de los años sesenta en plantaciones de tomate y pimienta (McManus et al., 2002). Pero el caso más grave de resistencia a este antibiótico, es el de Erwinia amylovora, ya que no se disponen de medidas de control alternativas que brinden resultados óptimos (Llacer et al., 2000; McGhee y Sundin, 2011). Se han encontrado genes en plásmidos conjuntivos que están relacionados con la resistencia a estreptomicina en aislamientos de Xanthomonas sp., Erwinia sp. y Pseudomonas sp. (Llop et al., 2012; Behlau et al., 2012). Otros casos de resistencia a productos como kasugamicina y validamicina 
han sido reportados (Ishikawa et al., 2004; Oliveira et al., 2007; Adaskaveg et al., 2008). Antibióticos como ampicilina y cloranfenicol, han sido poco empleados para el control de enfermedades fitosanitarias, pero al tener un amplio espectro de acción, están cobrando fuerza como agentes de control de bacteriosis en plantas ornamentales, con un alto riesgo pues son de uso en humanos, generando a futuro problemas serios de resistencia, por tanto su uso debe ser experimental, mas no extendido en agricultura (Pérez, 1994).

El cobre es uno de los bactericidas más utilizados en todo el mundo, pero diversos experimentos han demostrado la existencia de cepas resistentes a concentraciones bajas de este producto (Quezado et al., 2003; Martin et al., 2004; Maneguim et al., 2007; Behlau et al., 2012). Behlau et al. (2012) afirman que, dependiendo de la cepa, hay presencia de genes cop de resistencia a cobre: $c o p L, c o p A$, $\operatorname{cop} \mathrm{B}, \operatorname{cop} \mathrm{M}, \operatorname{cop} \mathrm{G}, \operatorname{cop} \mathrm{C}, \operatorname{cop} \mathrm{D}, \mathrm{y} \operatorname{cop} \mathrm{F}, \mathrm{y}$ pueden estar localizados en plásmidos o en el cromosoma.

Debido al eventual uso de antibióticos en plantaciones de pasifloras, el presente estudio tuvo como objetivo evaluar la sensibilidad a algunas de estas moléculas y productos cúpricos de uso agrícola en condiciones de laboratorio, utilizando 111 aislamientos bacterianos provenientes de cultivos de gulupa y maracuyá de diferentes localidades de Colombia con sintomatología de bacteriosis.

\section{MATERIALES Y MÉTODOS}

\section{Material biológico}

Se emplearon 111 aislamientos bacterianos, provenientes del cepario del laboratorio de Sanidad Vegetal de la Facultad de Agronomía de la Universidad Nacional, Bogotá, provenientes de bacterias asociadas a síntomas de bacteriosis en cultivos gulupa ( $P$. edulis) y maracuyá ( $P$. edulis var. flavicarpa) (tabla 1), ubicados en diferentes regiones de Colombia como los departamentos de Cundinamarca, Huila, Valle del Cauca, Santan- der, Antioquia, Boyacá, Tolima, Meta, Risaralda y Caldas. Los aislamientos fueron identificados mediante pruebas bioquímicas especializadas, pruebas moleculares, y mantenidas en glicerol $15 \%$ a $-20^{\circ} \mathrm{C}$; se sembraron en agar nutritivo (OXOID) (AN) a $28^{\circ} \mathrm{C}$ cuando se requirieron.

\section{Evaluación de la sensibilidad a antibióticos y productos cúpricos}

La sensibilidad in vitro, se determinó mediante el método modificado de incorporación en agar descrito por Quezado et al. (2003), suplementando AN con el antibiótico a evaluar. Los experimentos fueron realizados con diferentes grupos de antibióticos, aminoglucósidos (estreptomicina: $25 \mathrm{mg} \mathrm{L}^{-1}$, sulfato de kanamicina: 25 $\mathrm{mg} \mathrm{L}^{-1}$, kasugamicina: $200 \mathrm{mg} \mathrm{L}^{-1}$ ), tetraciclinas (oxitetraciclina: $25 \mathrm{mg} \mathrm{L}^{-1}$ ), penicilina (ampicilina: $50 \mathrm{mg} \mathrm{L}^{-1}$ ), fenicol (cloranfenicol: $50 \mathrm{mg} \mathrm{L}^{-1}$ ) y validamicina (validamicina: $200 \mathrm{mg} \mathrm{L}^{-1}$ ). Para preparar las suspensiones bacterianas, fueron empleadas colonias de cada uno de los aislamientos, incubadas durante $48 \mathrm{~h}$ a $28^{\circ} \mathrm{C}$, y resuspendidas en agua destilada estéril hasta obtener una concentración de $10^{7} \mathrm{ufc} / \mathrm{mL}$. La siembra de las suspensiones se realizó en cámara de flujo horizontal Streamline ${ }^{\circledR}$, añadiendo alícuotas de 10 $\mu \mathrm{L}$ de la suspensión bacteriana en seis puntos distintos del medio suplementado con el antibiótico. Cada uno de los aislamientos fue sembrado de la forma anteriormente descrita y luego de la siembra, se esperó la incorporación de las gotas al medio, y fueron incubadas por $48 \mathrm{~h}$ a $28^{\circ} \mathrm{C}$. Como control, se sembraron las mismas suspensiones bacterianas en cajas de Petri con AN no suplementado, e incubadas bajo las mismas condiciones.

La sensibilidad in vitro a productos cúpricos de los 111 aislamientos, se evaluó mediante la metodología empleada por Loper et al. (1991) y Cooksey et al. (1990), suplementando AN con oxicloruro de cobre y sulfato de cobre a una concentración final de $750 \mathrm{mg} \mathrm{L}^{-1}$, y la metodología de siembra fue similar a la descrita para medios con antibióticos. 
Tabla 1. Información general de los aislamientos bacterianos asociados a pasifloras empleados en el estudio.

\begin{tabular}{|c|c|c|c|c|c|c|c|}
\hline Consecutivo & Especie vegetal & $\begin{array}{c}\text { Órgano de } \\
\text { la planta }\end{array}$ & Departamento & Municipio & $\begin{array}{l}\text { Altitud } \\
\text { (msnm) }\end{array}$ & $\begin{array}{l}\text { Tempera- } \\
\text { tura }\left({ }^{\circ} \mathrm{C}\right)\end{array}$ & Género presuntivo \\
\hline FAB001 & P. edulis & Hoja & Cundinamarca & Venecia & 1.423 & 18 & Erwinia spp. \\
\hline FAB002 & P. edulis & Ноја & Cundinamarca & Venecia & 1.423 & 18 & Erwinia spp. \\
\hline FAB003 & P. edulis & Hoja & Cundinamarca & Venecia & 1.423 & 18 & Erwinia spp. \\
\hline FAB005 & P. edulis & Hoja & Cundinamarca & Venecia & 1.423 & 18 & Burkholderia spp. \\
\hline FAB006 & P. edulis & Hoja & Cundinamarca & Venecia & 1.423 & 18 & Pantoea spp. \\
\hline FAB008 & P. edulis & Hoja & Cundinamarca & Venecia & 1.423 & 18 & Pseudomonas spp. \\
\hline FAB009 & P. edulis & Hoja & Cundinamarca & Venecia & 1.423 & 18 & Pseudomonas spp. \\
\hline FAB011 & P. edulis & Hoja & Cundinamarca & Silvania & 1.470 & 20 & Erwinia spp. \\
\hline FAB013 & P. edulis & Hoja & Cundinamarca & Silvania & 1.470 & 20 & Pantoea spp. \\
\hline FAB015 & P. edulis & Hoja & Cundinamarca & Silvania & 1.470 & 20 & Burkholderia spp. \\
\hline FAB016 & P. edulis & Hoja & Cundinamarca & Venecia & 1.423 & 18 & Pantoea spp. \\
\hline FAB017 & P. edulis & Hoja & Cundinamarca & Venecia & 1.423 & 18 & Pantoea spp. \\
\hline FAB018 & P. edulis & Fruto & Cundinamarca & Venecia & 1.423 & 18 & Pantoea spp. \\
\hline FAB020 & P. edulis & Fruto & Cundinamarca & Venecia & 1.423 & 18 & Pantoea spp. \\
\hline FAB021 & P. edulis & Hoja & Cundinamarca & Venecia & 1.423 & 18 & Pantoea spp. \\
\hline FAB025 & P. edulis & Hoja & Cundinamarca & Granada & 1.890 & 14 & $\begin{array}{l}\text { Endófito no } \\
\text { identificado }\end{array}$ \\
\hline FAB026 & P. edulis & Hoja & Cundinamarca & Granada & 1.890 & 14 & $\begin{array}{l}\text { Endófito no } \\
\text { identificado }\end{array}$ \\
\hline FAB027 & P. edulis & Fruto & Cundinamarca & Granada & 1.890 & 14 & $\begin{array}{l}\text { Endófito no } \\
\text { identificado }\end{array}$ \\
\hline FAB028 & P. edulis & Hoja & Cundinamarca & Granada & 1.890 & 14 & Pantoea spp. \\
\hline FAB030 & P. edulis & Hoja & Cundinamarca & Granada & 1.890 & 14 & Erwinia spp. \\
\hline FAB031 & P. edulis & Hoja & Cundinamarca & Granada & 1.890 & 14 & $\begin{array}{l}\text { Endófito no } \\
\text { identificado }\end{array}$ \\
\hline FAB032 & P. edulis & Tallo & Cundinamarca & Granada & 1.890 & 14 & Erwinia spp. \\
\hline FAB034 & P. edulis & Fruto & Cundinamarca & Granada & 1.890 & 14 & Erwinia spp. \\
\hline FAB035 & P. edulis & Hoja & Cundinamarca & Granada & 1.890 & 14 & Burkholderia spp. \\
\hline FAB037 & P. edulis & Hoja & Cundinamarca & Granada & 1.890 & 14 & $\begin{array}{l}\text { Endófito no } \\
\text { identificado }\end{array}$ \\
\hline FAB040 & P. edulis & Fruto & Cundinamarca & Granada & 1.890 & 14 & Pantoea spp. \\
\hline FAB041 & P. edulis & Hoja & Cundinamarca & Granada & 1.890 & 14 & Erwinia spp. \\
\hline FAB042 & P. edulis & Hoja & Cundinamarca & Granada & 1.890 & 14 & Burkholderia spp. \\
\hline FAB043 & P. edulis & Hoja & Cundinamarca & Granada & 1.890 & 14 & $\begin{array}{l}\text { Endófito no } \\
\text { identificado }\end{array}$ \\
\hline FAB045 & P. edulis & Tallo & Cundinamarca & Granada & 1.890 & 14 & Xanthomonas spp. \\
\hline FAB047 & P. edulis & Tallo & Cundinamarca & Granada & 1.890 & 14 & Xanthomonas spp. \\
\hline FAB048 & P. edulis & Hoja & Cundinamarca & Granada & 1.890 & 14 & Burkholderia spp. \\
\hline FAB049 & P. edulis & Hoja & Cundinamarca & Granada & 1.890 & 14 & Erwinia spp. \\
\hline
\end{tabular}




\begin{tabular}{|c|c|c|c|c|c|c|c|}
\hline Consecutivo & Especie vegetal & $\begin{array}{c}\text { Órgano de } \\
\text { la planta }\end{array}$ & Departamento & Municipio & $\begin{array}{l}\text { Altitud } \\
\text { (msnm) }\end{array}$ & $\begin{array}{l}\text { Tempera- } \\
\text { tura }\left({ }^{\circ} \mathrm{C}\right)\end{array}$ & Género presuntivo \\
\hline FAB050 & P. edulis & Hoja & Cundinamarca & Granada & 1.890 & 14 & Erwinia spp. \\
\hline FAB051 & P. edulis & Hoja & Cundinamarca & Granada & 1.890 & 14 & Erwinia spp. \\
\hline FAB052 & P. edulis & Hoja & Cundinamarca & Granada & 1.890 & 14 & Xanthomonas spp. \\
\hline FAB053 & P. edulis & Hoja & Cundinamarca & Granada & 1.890 & 14 & Xanthomonas spp. \\
\hline FAB054 & P. edulis & Fruto & Cundinamarca & Granada & 1.890 & 14 & Xanthomonas spp. \\
\hline FAB055 & P. edulis & Fruto & Cundinamarca & Granada & 1.890 & 14 & Erwinia spp. \\
\hline FAB056 & P. edulis & Fruto & Cundinamarca & Granada & 1.890 & 14 & Erwinia spp. \\
\hline FAB057 & P. edulis & Fruto & Cundinamarca & Granada & 1.890 & 14 & Erwinia spp. \\
\hline FAB058 & P. edulis & Fruto & Cundinamarca & Granada & 1.890 & 14 & Erwinia spp. \\
\hline FAB059 & P. edulis & Fruto & Cundinamarca & Granada & 1.890 & 14 & Erwinia spp. \\
\hline FAB060 & P. edulis & $\begin{array}{l}\text { Tallo } \\
\text { central }\end{array}$ & Cundinamarca & Venecia & 1.423 & 18 & Erwinia spp. \\
\hline FAB062 & P. edulis & $\begin{array}{l}\text { Tallo } \\
\text { central } \\
\end{array}$ & Cundinamarca & Venecia & 1.423 & 18 & $\begin{array}{l}\text { Endófito no } \\
\text { identificado }\end{array}$ \\
\hline FAB063 & P. edulis & Fruto & Boyacá & Buena Vista & 1.984 & 18 & Erwinia spp. \\
\hline FAB064 & P. edulis & Fruto & Boyacá & Buena Vista & 1.984 & 18 & Erwinia spp. \\
\hline FAB065 & P. edulis & Hoja & Boyacá & Buena Vista & 1.984 & 18 & Erwinia spp. \\
\hline FAB066 & P. edulis & Hoja & Boyacá & Buena Vista & 1.984 & 18 & Erwinia spp. \\
\hline FAB067 & P. edulis & Hoja & Boyacá & Buena Vista & 1.984 & 18 & Pseudomonas spp. \\
\hline FAB068 & P. edulis & Hoja & Boyacá & Buena Vista & 1.984 & 18 & Erwinia spp. \\
\hline FAB069 & P. edulis & Fruto & Boyacá & Buena Vista & 1.984 & 18 & Pantoea spp. \\
\hline FAB070 & P. edulis & Fruto & Boyacá & Buena Vista & 1.984 & 18 & $\begin{array}{l}\text { Endófito no } \\
\text { identificado }\end{array}$ \\
\hline FAB071 & P. edulis & Fruto & Boyacá & Buena Vista & 1.984 & 18 & Xanthomonas spp. \\
\hline FAB072 & P. edulis & $\begin{array}{l}\text { Tallo } \\
\text { central }\end{array}$ & Boyacá & Buena Vista & 1.984 & 18 & Erwinia spp. \\
\hline FAB073 & P. edulis & $\begin{array}{l}\text { Tallo } \\
\text { central }\end{array}$ & Boyacá & Buena Vista & 1.984 & 18 & Xanthomonas spp. \\
\hline FAB074 & P. edulis & Fruto & Cundinamarca & Granada & 1.890 & 14 & Erwinia spp. \\
\hline FAB075 & P. edulis & Fruto & Cundinamarca & Granada & 1.890 & 14 & Pseudomonas spp. \\
\hline FAB076 & P. edulis & Hoja & Cundinamarca & $\begin{array}{l}\text { San } \\
\text { Bernardo }\end{array}$ & 1.600 & 20 & Erwinia spp. \\
\hline FAB077 & P. edulis & Hoja & Huila & La Argentina & 2.408 & 18 & Erwinia spp. \\
\hline FAB078 & P. edulis & Fruto & Huila & La Argentina & 2.408 & 18 & Xanthomonas spp. \\
\hline FAB079 & P. edulis & Tallo & Huila & La Argentina & 2.408 & 18 & Erwinia spp. \\
\hline FAB080 & P. edulis & Fruto & Cundinamarca & $\begin{array}{l}\text { San } \\
\text { Bernardo }\end{array}$ & 1.600 & 20 & $\begin{array}{l}\text { Endófito no } \\
\text { identificado }\end{array}$ \\
\hline FAB081 & P. edulis & Fruto & Cundinamarca & $\begin{array}{l}\text { San } \\
\text { Bernardo }\end{array}$ & 1.600 & 20 & $\begin{array}{l}\text { Endófito no } \\
\text { identificado }\end{array}$ \\
\hline FAB082 & P. edulis & Hoja & Huila & La Argentina & 2.408 & 18 & Erwinia spp. \\
\hline FAB083 & P. edulis & Hoja & Huila & Gigante & 860 & 24 & Erwinia spp. \\
\hline
\end{tabular}




\begin{tabular}{|c|c|c|c|c|c|c|c|}
\hline Consecutivo & Especie vegetal & $\begin{array}{l}\text { Órgano de } \\
\text { la planta }\end{array}$ & Departamento & Municipio & $\begin{array}{l}\text { Altitud } \\
\text { (msnm) }\end{array}$ & $\begin{array}{l}\text { Tempera- } \\
\text { tura }\left({ }^{\circ} \mathrm{C}\right)\end{array}$ & Género presuntivo \\
\hline FAB085 & P. edulis & Hoja & Antioquia & Peñol & 2.000 & 17 & Erwinia spp. \\
\hline FAB086 & P. edulis & Fruto & Tolima & Cajamarca & 1.814 & 19 & Erwinia spp. \\
\hline FAB087 & P. edulis & Fruto & Tolima & Cajamarca & 1.814 & 19 & Xanthomonas spp. \\
\hline FAB088 & P. edulis & Hoja & Tolima & Cajamarca & 1.814 & 19 & Xanthomonas spp. \\
\hline FAB089 & P. edulis & Fruto & Antioquia & Peñol & 2.000 & 17 & Erwinia spp. \\
\hline FAB090 & P. edulis & Tallo & Tolima & Cajamarca & 1.814 & 19 & Pseudomonas spp. \\
\hline FAB091 & P. edulis & Hoja & Cundinamarca & Granada & 1.890 & 14 & Xanthomonas spp. \\
\hline FAB092 & P. edulis & Hoja & Cundinamarca & Granada & 1.890 & 14 & Erwinia spp. \\
\hline FAB093 & P. edulis & Hoja & Cundinamarca & Granada & 1.890 & 14 & Erwinia spp. \\
\hline FAB094 & $\begin{array}{l}\text { P. edulis var. } \\
\text { flavicarpa }\end{array}$ & Fruto & Meta & San Martín & 420 & 27 & Erwinia spp. \\
\hline FAB095 & $\begin{array}{l}\text { P. edulis var. } \\
\text { flavicarpa }\end{array}$ & Fruto & Meta & San Martín & 420 & 27 & Erwinia spp. \\
\hline FAB096 & P. edulis & Fruto & Cundinamarca & Granada & 1.890 & 14 & Erwinia spp. \\
\hline FAB097 & P. edulis & Fruto & Cundinamarca & Granada & 1.890 & 14 & Erwinia spp. \\
\hline FAB098 & P. edulis & Fruto & Cundinamarca & Granada & 1.890 & 14 & Pseudomonas spp. \\
\hline FAB099 & P. edulis & Fruto & Cundinamarca & Granada & 1.890 & 14 & Pantoea spp. \\
\hline FAB100 & P. edulis & Fruto & Cundinamarca & Granada & 1.890 & 14 & Pseudomonas spp. \\
\hline FAB101 & P. edulis & Hoja & Cundinamarca & $\begin{array}{l}\text { San } \\
\text { Bernardo }\end{array}$ & 1.600 & 20 & Xanthomonas spp. \\
\hline FAB102 & P. edulis & Hoja & Cundinamarca & $\begin{array}{l}\text { San } \\
\text { Bernardo }\end{array}$ & 1.600 & 20 & Erwinia spp. \\
\hline FAB103 & P. edulis & Hoja & Cundinamarca & $\begin{array}{l}\text { San } \\
\text { Bernardo }\end{array}$ & 1.600 & 20 & Erwinia spp. \\
\hline FAB104 & P. edulis & Hoja & Cundinamarca & Venecia & 1.423 & 18 & Erwinia spp. \\
\hline FAB105 & P. edulis & Hoja & Cundinamarca & Venecia & 1.423 & 18 & Erwinia spp. \\
\hline FAB106 & P. edulis & Hoja & Tolima & Ibagué & 1.225 & 24 & $\begin{array}{l}\text { Endófito no } \\
\text { identificado }\end{array}$ \\
\hline FAB107 & P. edulis & Hoja & Tolima & Ibagué & 1.225 & 24 & Erwinia spp. \\
\hline FAB108 & P. edulis & Fruto & Tolima & Ibagué & 1.225 & 24 & Erwinia spp. \\
\hline FAB109 & P. edulis & Fruto & Tolima & Ibagué & 1.225 & 24 & Xanthomonas spp. \\
\hline FAB110 & P. edulis & Fruto & Tolima & Ibagué & 1.225 & 24 & Xanthomonas spp. \\
\hline FAB111 & $\begin{array}{l}\text { P. edulis var. } \\
\text { flavicarpa }\end{array}$ & Hoja & Valle del Cauca & Pradera & 1.070 & 23 & Erwinia spp. \\
\hline FAB112 & P. edulis & Fruto & Caldas & Manizales & 2.150 & 18 & Erwinia spp. \\
\hline FAB113 & P. edulis & Fruto & Caldas & Manizales & 2.150 & 18 & Erwinia spp. \\
\hline FAB114 & P. edulis & Hoja & Caldas & Manizales & 2.150 & 18 & Erwinia spp. \\
\hline FAB116 & P. edulis & Hoja & Risaralda & Manizales & 2.150 & 18 & $\begin{array}{l}\text { Endófito no } \\
\text { identificado }\end{array}$ \\
\hline FAB117 & P.quadrangularis & Hoja & Risaralda & $\begin{array}{l}\text { Sta. Rosa } \\
\text { de Cabal }\end{array}$ & 1.701 & 19 & Erwinia spp. \\
\hline
\end{tabular}




\begin{tabular}{|c|c|c|c|c|c|c|c|}
\hline Consecutivo & Especie vegetal & $\begin{array}{c}\text { Órgano de } \\
\text { la planta }\end{array}$ & Departamento & Municipio & $\begin{array}{c}\text { Altitud } \\
\text { (msnm) }\end{array}$ & $\begin{array}{l}\text { Tempera- } \\
\text { tura }\left({ }^{\circ} \mathrm{C}\right)\end{array}$ & Género presuntivo \\
\hline FAB118 & P.quadrangularis & Hoja & Risaralda & $\begin{array}{l}\text { Sta. Rosa } \\
\text { de Cabal }\end{array}$ & 1.701 & 19 & Erwinia spp. \\
\hline FAB119 & $\begin{array}{l}\text { P. edulis var. } \\
\text { flavicarpa }\end{array}$ & Fruto & Valle del Cauca & $\begin{array}{l}\text { Sta. Rosa } \\
\text { de Cabal }\end{array}$ & 1.701 & 19 & $\begin{array}{l}\text { Endófito no } \\
\text { identificado }\end{array}$ \\
\hline FAB120 & $\begin{array}{l}\text { P. edulis var. } \\
\text { flavicarpa }\end{array}$ & Hoja & Valle del Cauca & Pradera & 1.070 & 23 & Xanthomonas spp. \\
\hline FAB121 & $\begin{array}{l}\text { P. edulis var. } \\
\text { flavicarpa }\end{array}$ & Hoja & Valle del Cauca & Pradera & 1.070 & 23 & Erwinia spp. \\
\hline FAB122 & $\begin{array}{l}\text { P. edulis var. } \\
\text { flavicarpa }\end{array}$ & Fruto & Valle del Cauca & Pradera & 1.070 & 23 & $\begin{array}{l}\text { Endófito no } \\
\text { identificado }\end{array}$ \\
\hline FAB123 & $\begin{array}{l}\text { P. edulis var. } \\
\text { flavicarpa }\end{array}$ & Hoja & Valle del Cauca & La Unión & 938 & 24 & Pantoea spp. \\
\hline FAB124 & $\begin{array}{l}\text { P. edulis var. } \\
\text { flavicarpa }\end{array}$ & Hoja & Valle del Cauca & La Unión & 938 & 24 & Erwinia spp.. \\
\hline FAB125 & $\begin{array}{l}\text { P. edulis var. } \\
\text { flavicarpa }\end{array}$ & Hoja & Valle del Cauca & La Unión & 938 & 24 & Xanthomonas spp. \\
\hline FAB126 & $\begin{array}{l}\text { P. edulis var. } \\
\text { flavicarpa }\end{array}$ & Hoja & Valle del Cauca & La Unión & 938 & 24 & Erwinia spp. \\
\hline FAB127 & $\begin{array}{l}\text { P. edulis var. } \\
\text { flavicarpa }\end{array}$ & Hoja & Valle del Cauca & La Unión & 938 & 24 & Erwinia spp. \\
\hline FAB128 & $\begin{array}{l}\text { P. edulis var. } \\
\text { flavicarpa }\end{array}$ & Hoja & Valle del Cauca & Toro & 944 & 23 & Xanthomonas spp. \\
\hline FAB129 & $\begin{array}{l}\text { P. edulis var. } \\
\text { flavicarpa }\end{array}$ & Hoja & Valle del Cauca & Toro & 944 & 23 & Xanthomonas spp. \\
\hline FAB130 & $\begin{array}{l}\text { P. edulis var. } \\
\text { flavicarpa }\end{array}$ & Hoja & Valle del Cauca & Roldanillo & 938 & 23 & Xanthomonas spp. \\
\hline
\end{tabular}

Transcurrido el tiempo de incubación, se observó la presencia o ausencia de crecimiento bacteriano en los seis puntos de siembra, considerándose resistentes los aislamientos que presentaron crecimiento confluente sobre la superficie del medio en las seis repeticiones.

Esto permitió determinar el número de aislamientos sensibles a los diferentes productos que fueron expuestos, además de su correlación con el porcentaje de aislamientos resistentes para cada antibiótico y producto cúprico evaluado.

\section{RESULTADOS Y DISCUSIÓN}

Posterior a la incubación del material biológico en los medios suplementados, se observó que los aislamientos sensibles a los antibióticos y pro- ductos cúpricos fueron incapaces de crecer sobre la superficie del medio, contrario a lo observado en los aislamientos resistentes. Cada uno de los aislamientos sembrados, fue comparado con su respectivo control.

Esto indica que el método de incorporación de los materiales en el agar empleado permitió la evaluación efectiva de los diferentes productos, ya que no se presentó inactivación de ninguno de estos y se observó con claridad la respuesta de los aislamientos a los productos evaluados. Adicionalmente, con esta metodología se logró determinar la eficacia de los antibióticos o productos cúpricos para inhibir o destruir a los microorganismos, atravesando las barreras superficiales de las bacterias y después fijándose sobre su respectivo sitio de acción. Por el contrario, en los aislamientos resistentes, a través de diferentes mecanismos 
de resistencia como la inhibición enzimática de antibiótico, la impermeabilidad de la membrana y la modificación de rutas metabólicas (mediante cambio de moléculas o sitios de acción específicos del antibiótico), pueden repercutir en la efectividad de los diferentes productos evaluados (Pérez, 1998; Balaban et al., 2004).

Wiuff et al. (2005) usando Escherichia coli CAB1 como modelo, y exponiéndola a antibióticos de cinco clases diferentes (ampicilina, ciprofloxacina, rifampicina, estreptomicina y tetraciclina), proponen que en un medio, puede suponerse que se presentan fenómenos como la descomposición de antibióticos, la resistencia heredada y la resistencia fenotípica, siendo estas últimas dos fenómenos existentes, demostrando que las subpoblaciones resistentes generadas por la exposición a una concentración de un antibiótico (por ejemplo constantes exposiciones en campo) también son resistentes a concentraciones más altas del mismo antibiótico y pueden tener resistencia cruzada a otros antibióticos.

\section{Sensibilidad de aislamientos bacterianos a antibióticos}

Algunos de los antibióticos inhibieron el crecimiento en agar sobre la totalidad de los aislamientos evaluados (tabla 2), que incluyeron géneros como Burkholderia sp., Erwinia sp., Pantoea sp., Pseudomonas sp., Xanthomonas sp. Los resultados indican que el tratamiento con cloranfenicol -fenicol- a $50 \mathrm{mg} \mathrm{L}^{-1}$ presentó el mayor porcentaje de inhibición (83\%) sobre los aislamientos probados. Si se analizan los resultados a partir de las concentraciones empleadas, el sulfato de kanamicina y sulfato de estreptomicina evaluados a $25 \mathrm{mg} \mathrm{L}^{-1}$, presentan porcentajes de control alto, $83 \%$ y $87 \%$, respectivamente.

Con kasugamicina (200 mg L $\mathrm{L}^{-1}$ ), se observó un control en el 50,4\% de los aislamientos bacterianos evaluados. Los menores índices de sensibilidad se observaron con ampicilina $(50 \mathrm{mg}$ $\left.\mathrm{L}^{-1}\right)$ y con validamicina $\left(200 \mathrm{mg} \mathrm{L}^{-1}\right)$, que pese a su alta concentración, solo inhibió aproximada-

Tabla 2. Evaluación in vitro de sensibilidad a antibióticos en 111 aislamientos bacterianos asociados a $P$. edulis

\begin{tabular}{|c|c|c|c|c|c|}
\hline $\begin{array}{l}\text { Producto } \\
\text { evaluado }\end{array}$ & $\begin{array}{c}\text { No. } \\
\text { aislamientos } \\
\text { sensibles }\end{array}$ & $\begin{array}{c}\text { Géneros bacterianos } \\
\text { sensibles }^{1}\end{array}$ & $\begin{array}{l}\text { No. } \\
\text { aislamientos } \\
\text { resistentes }\end{array}$ & $\begin{array}{l}\text { Porcentaje de } \\
\text { aislamientos } \\
\text { resistentes (\%) }\end{array}$ & $\begin{array}{l}\text { Géneros bacterianos } \\
\text { resistentes }\end{array}$ \\
\hline $\begin{array}{l}\text { Sulfato de } \\
\text { Kanamicina }\end{array}$ & 81 & $\begin{array}{l}4 \mathrm{Bk}, 40 \mathrm{Er}, 9 \mathrm{Pt}, 5 \mathrm{Ps}, \\
13 \mathrm{Xs}, 10 \mathrm{Nl} .\end{array}$ & 30 & 27 & $\begin{array}{l}1 \mathrm{Bk}, 14 \mathrm{Er}, 3 \mathrm{Pt}, 2 \mathrm{Ps}, 6 \\
\text { Xs, } 4 \mathrm{NI} .\end{array}$ \\
\hline $\begin{array}{l}\text { Sulfato de } \\
\text { Estreptomicina }\end{array}$ & 87 & $\begin{array}{l}5 \mathrm{Bk}, 44 \mathrm{Er}, 10 \mathrm{Pt}, 5 \mathrm{Ps}, \\
\mathrm{Xs}, 10 \mathrm{NI} .\end{array}$ & 23 & 23 & $\begin{array}{l}10 \text { Er, } 2 \text { Pt, } 2 \text { Ps, } 5 \text { Xs, } \\
4 \mathrm{NI} \text {. }\end{array}$ \\
\hline Cloranfenicol $^{2}$ & 92 & $\begin{array}{l}4 \mathrm{Bk}, 45 \mathrm{Er}, 11 \mathrm{Pt}, 7 \mathrm{Ps}, \\
16 \mathrm{Xs}, 9 \mathrm{Nl} .\end{array}$ & 19 & 17,1 & $\begin{array}{l}1 \mathrm{Bk}, 9 \mathrm{Er}, 1 \mathrm{Pt}, 3 \mathrm{Xs} \text {, } \\
5 \mathrm{Nl} .\end{array}$ \\
\hline Ampicilina $^{2}$ & 14 & $\begin{array}{l}1 \mathrm{Bk}, 7 \mathrm{Er}, 3 \mathrm{Pt}, 1 \mathrm{Ps} \text {, } \\
2 \mathrm{Xs.}\end{array}$ & 97 & 87,3 & $\begin{array}{l}4 \mathrm{Bk}, 47 \mathrm{Er}, 9 \mathrm{Pt}, 6 \mathrm{Ps} \text {, } \\
17 \mathrm{Xs}, 14 \mathrm{NI} .\end{array}$ \\
\hline Kasugamicina & 55 & $\begin{array}{l}4 \text { Bk, } 25 \text { Er, } 6 \text { Pt, } 5 \text { Ps, } 8 \\
\text { Xs, } 7 \text { NI. }\end{array}$ & 56 & 50,4 & $\begin{array}{l}1 \mathrm{Bk}, 29 \mathrm{Er}, 6 \mathrm{Pt}, 2 \mathrm{Ps}, \\
11 \mathrm{Xs}, 7 \mathrm{NI} .\end{array}$ \\
\hline Validamicina & 5 & $1 \mathrm{Er}, 1 \mathrm{Pt}, 1 \mathrm{Ps}, 2 \mathrm{NI}$. & 106 & 95,5 & $\begin{array}{l}5 \mathrm{Bk}, 53 \mathrm{Er}, 11 \mathrm{Pt}, 6 \mathrm{Ps} \text {, } \\
19 \mathrm{Xs}, 12 \mathrm{Nl}\end{array}$ \\
\hline Oxitetraciclina ${ }^{3}$ & 15 & 2Bk, 1Er, 1Pt, 3Ps, 8Xs. & 8 & 34,7 & 2Bk, 3Er, 1Pt, 2Xs. \\
\hline
\end{tabular}

$1 \quad$ Bk: Burkholderia sp.; Er: Erwinia sp.; Pt: Pantoea sp.; Ps: Pseudomonas sp.; Xs:Xanthomonas sp.; Nl: Endófitos no identificados.

2 Antibióticos restringidos para agricultura. Su uso en plantas representa riesgos altos en el ambiente y en salud humana.

3 Evaluación realizada en 23 aislamientos bacterianos. 
mente el 9\% de las bacterias evaluadas. La resistencia por parte de los aislamientos a dos de los aminoglucósidos evaluados, sulfato de kanamicina y kasugamicina, se puede relacionar con elementos y mecanismos de resistencia cromosómicos y extracromosómicos (Behlau et al., 2012); los primeros se deben a mutaciones en los loci, que conllevan a cambios en las proteínas ribosómicas en las que se fijan estos antibióticos. En el segundo mecanismo se dan elementos móviles de resistencia como los transposones y plásmidos, en donde se encuentran enzimas modificantes (como acetiltrasferasas, nucleotidiltransferasas y fosfotransferasas) que impiden la entrada del aminoglucósido a través de la membrana celular (Dámaso, 1990). Por otra parte, se ha reportado efectividad del control in vitro de kasugamicina sobre aislamientos bacterianos fitopatógenos a concentraciones superiores a las empleadas en este trabajo (500 y 700 $\mathrm{mg} \mathrm{L}^{-1}$ ) (Sales et al., 2005). Oliveira et al. (2007) reportaron que a concentraciones de $87 \mathrm{mg} \mathrm{L}^{-1}$, este producto no ejerce control sobre bacterias fitopatógenas.

Un alto número de aislamientos fueron sensibles al sulfato de estreptomicina a $25 \mathrm{mg} \mathrm{L}^{-1}$, con la concentración más baja empleada en la presente investigación (tabla 2). La estreptomicina es uno de los antibióticos más empleados en la agricultura mundial desde hace más de cinco décadas, y para el cual se han reportado cepas resistentes desde los años sesenta (McManus et al., 2002). Los resultados en este trabajo son contrarios a los de Quezado et al. (2003), quienes obtuvieron un $60 \%$ de aislamientos de Xanthomonas sp. resistentes a sulfato de estreptomicina a $25 \mathrm{mg} \mathrm{L}^{-1}$, afirmando que la resistencia se expresa solo en los niveles bajos de concentración. Sin embargo, algunos autores mencionan resistencia a concentraciones mayores, no solo en especies de Xanthomonas sp., sino también de Pseudomonas sp. y E. amylovora (Misavage et al., 1990; Spotts y Cervantes, 1995; Russo et al., 2008). Por otra parte, en este trabajo fueron analizadas cepas bacterianas pa- togénicas y no patogénicas, similar a lo realizado por Behlau et al. (2012), quienes realizando aplicaciones de estreptomicina en plantaciones de cítricos afectadas por $X$. citri encontraron un significativo aumento de bacterias epíficas resistentes o resistentes a este antibiótico en el transcurso del tiempo, debido a la presión de selección para la resistencia bacteriana. Esto, a la larga, representa un riesgo en agricultura por fenómenos de transmisión horizontal de elementos génicos que puedan conferir resistencia.

En el caso de los resultados con el cloranfenicol, se obtuvo una gran proporción de aislamientos sensibles (83\%). Similar a lo obtenido por Shenge et al. (2007) para Xanthomonas campestris pv. vesicatoria en Tanzania, con un $77 \%$ de sensibilidad a una concentración de $20 \mathrm{mg}$ $\mathrm{L}^{-1}$ del antibiótico. Con relación a los aislamientos resistentes a este antibiótico, es posible que se deba a impermeabilidad de las células bacterianas al cloranfenicol (resistencia cromosómica), o sean portadores de elementos móviles de resistencia extracromosómica, como el Factor $\mathrm{R}$ (Chinedum, 2005). Sin embargo, es necesario aclarar que este es un antibiótico de uso humano, y tal como se ha advertido hace varios años, no debe ser usado en agricultura bajo ningún escenario. El uso indiscriminado del cloranfenicol en agricultura, normaliza poblaciones bacterianas con plásmidos de resistencia, siendo esto de alto riesgo en salud humana, pues inutiliza antibióticos de amplio espectro (Khachatourians, 1998).

La validamicina y la ampicilina presentaron los menores índices de sensibilidad contrario a lo reportado por Shenge et al. (2007), quienes obtuvieron sensibilidad a ampicilina (50 $\left.\mathrm{mg} \mathrm{L}^{-1}\right)$ de todos los aislamientos evaluados. Los altos niveles de resistencia resultantes para ambos antibióticos pueden estar relacionados con resistencia natural. Los aislamientos evaluados podrían tener esta propiedad intrínseca, que les permite no ser afectados por los fármacos, esto puede ser debido al contacto con concen- 
traciones remanentes de los antibióticos ya que se encuentran de manera residual en los suelos (Thiele-Bruhn, 2003). Esta situación ya ha sido reportada para validamicina por Ishikawa et al. (2004), quienes no inhibieron a $X$. campestris pv. campestris, en medios suplementados con una concentración de $10 \mathrm{mg} \mathrm{L}^{-1}$ del antibiótico, aun empleando concentraciones superiores a $50 \mathrm{mg}$ $\mathrm{L}^{-1}$ en el presente trabajo (200 $\left.\mathrm{mg} \mathrm{L}^{-1}\right)$, la mayoría de los aislamientos presentan resistencia al antibiótico.

Para el caso de oxitetraciclina, se obtuvo resistencia con ocho de los 23 aislamientos evaluados. Estos resultados son contradictorios a los reportados por Quezado et al. (2003) quienes no obtuvieron aislamientos resistentes a clorhidrato de oxitetraciclina a $25 \mathrm{mg} \mathrm{L}^{-1}$. Por otro lado, existen reportes de aislamientos de E. amylovora (McManus et al., 2002) y Pseudomonas sp. resistentes a oxitetraciclina en concentraciones de mayores a $50 \mathrm{mg} \mathrm{L}^{-1}$ (Spotts y Cervantes, 1995; Zang et al., 2012).

\section{Determinación de sensibilidad de los aislamientos, de acuerdo con el número de antibióticos evaluados}

El aislamiento de Erwinia sp. FAB095 (San Martín, Meta) (tabla 1) fue tolerante a seis de los antibióticos evaluados. Treinta y siete aislamientos $(31 \%)$ presentaron sensibilidad a cuatro antibióticos de forma compartida. A tres de seis antibióticos, fue sensible el $25 \%$ de la población, seguido por un $18 \%$ y $13 \%$ que fueron sensibles a dos y uno de los antibióticos, respectivamente. Posiblemente la resistencia a múltiples antibióticos (resistencia cruzada) podría estar respaldada por el desarrollo de mecanismos de supervivencia eficaces ante diferentes tipos de principios activos de antibióticos. Este término se relaciona con los antibióticos de estructura química análoga, ya que si el mecanismo de acción del antibiótico es similar y la bacteria es resistente a uno de los integrantes del grupo, lo será también para los demás (Chi- nedum, 2005). Lo que explicaría en este trabajo, la existencia de aislamientos resistentes de forma simultánea a los tres aminoglucósidos evaluados (sulfato de estreptomicina, kanamicina y kasugamicina), esto indicaría la posibilidad de que algunos de los aislamientos evaluados aquí pueden llevar consigo varios elementos que confieren resistencia a múltiples antibióticos, sin embargo, para que esto sea demostrado, se deben realizar análisis moleculares que indiquen la presencia de elementos cromósomicos o extracromosómicos para los diferentes productos evaluados.

\section{Sensibilidad de los aislamientos por procedencia de origen}

En los principales departamentos productores de pasifloráceas en Colombia (Boyacá, Cundinamarca, Meta y Tolima) se observaron aislamientos resistentes a todos los productos evaluados (tabla 3), estos resultados son el primer reporte de sensibilidad a antibióticos y productos cúpricos de bacterias asociadas a bacteriosis en plantas de gulupa del país.

Para la región del Valle del Cauca se obtuvo resistencia a estreptomicina por parte de tres aislamientos de Xanthomonas sp., a diferencia de lo encontrado por Castillo y Granada (1995), quienes obtuvieron la mayor eficacia de control de $X$. campestris pv. passiflorae en plantas de maracuyá, con sulfato de estreptomicina e hidróxido de cobre a concentraciones de $1.000 \mathrm{mg} \mathrm{L}^{-1} \mathrm{y}$ $3.000 \mathrm{mg} \mathrm{L}^{-1}$, respectivamente. Con kasugamicina, estos autores, al igual que en este trabajo, no obtuvieron un control adecuado de la enfermedad.

Actualmente, el control de bacteriosis en cultivos de gulupa en Colombia se realiza con kasugamicina y productos a base de cobre (Angulo, 2009), por lo cual los índices de resistencia presentados para estos productos, se encuentran relacionados con las aplicaciones realizadas en dichos departamentos. Además, el empleo de 
Tabla 3. Aislamientos asociados a $P$. edulis con pérdida de sensibilidad a antibióticos por región productora en Colombia.

\begin{tabular}{|c|c|c|c|c|c|c|c|c|}
\hline \multirow{2}{*}{ Departamento } & \multirow{2}{*}{$\begin{array}{c}\text { No. de } \\
\text { aislamientos }\end{array}$} & \multicolumn{7}{|c|}{ No. aislamientos resistentes a diferentes antibióticos* } \\
\hline & & $\mathrm{SK}^{1}$ & $\mathrm{SE}^{2}$ & $\mathrm{CL}^{3}$ & $\mathrm{AM}^{4}$ & $\mathrm{KA}^{5}$ & $V^{6}{ }^{6}$ & OC \\
\hline Antioquia & 2 & 0 & 0 & 0 & 2 & 1 & 2 & 2 \\
\hline Boyacá & 11 & 2 & 1 & 2 & 11 & 5 & 9 & 11 \\
\hline Caldas & 4 & 0 & 0 & 1 & 2 & 2 & 4 & 4 \\
\hline Cundinamarca & 62 & 10 & 8 & 11 & 52 & 26 & 59 & 62 \\
\hline Huila & 5 & 1 & 0 & 2 & 5 & 3 & 5 & 5 \\
\hline Meta & 2 & 2 & 2 & 1 & 2 & 2 & 2 & 2 \\
\hline Risaralda & 3 & 2 & 0 & 1 & 3 & 3 & 3 & 3 \\
\hline Tolima & 9 & 5 & 4 & 1 & 9 & 6 & 9 & 9 \\
\hline Valle del Cauca & 13 & 8 & 8 & 0 & 11 & 8 & 13 & 13 \\
\hline Total & 111 & 30 & 23 & 19 & 97 & 56 & 106 & 111 \\
\hline
\end{tabular}

*SK: Sulfato de Kanamicina, SE: Sulfato de Estreptomicina CL: Cloranfenicol AM: Ampicilina K: Kasugamicina VA: Validamicina OC: Oxitetraciclina

sulfato de kanamicina, cloranfenicol y ampicilina, en cultivos de esta pasiflorácea no ha sido reportado oficialmente, por tanto, los índices de resistencia a estos productos en los nueve departamentos, posiblemente se relacionarían con resistencia natural o adquirida (cromosómica o extracromosómica), como se explicó anteriormente.

En cuanto a los datos obtenidos para kasugamicina, Cundinamarca y Valle del Cauca (tabla 3), presentaron el mayor número de aislamientos resistentes a este antibiótico 26 y 8 , respectivamente en contraste con lo reportado en Brasil por Oliveira et al. (2007), quienes obtuvieron resistencia de la totalidad de los aislamientos provenientes de dos poblaciones del norte de del país. Estudios de sensibilidad de bacterias fitopatógenas de varias áreas del Brasil también indicaron que los huertos de manzano que habían recibido numerosos tratamientos con estreptomicina tenían una mayor proporción de aislamientos resistentes, mientras que los huertos que habían recibido pocos o ningún tratamiento con estreptomicina, no presentaban aislamientos resistentes (Cooksey, 1990).
Esto podría indicar que en aquellas regiones de Colombia, donde todos los aislamientos fueron sensibles a los antibióticos (Antioquia, Caldas, Huila y Risaralda), los productores no hacen uso generalizado de estos productos, por lo tanto las aplicaciones realizadas hasta el momento no han generado un efecto negativo (resistencia) en las poblaciones de bacterias.

\section{Sensibilidad de aislamientos bacterianos a productos cúpricos}

El sulfato de cobre ejerció control sobre toda la población evaluada con la concentración de 750 $\mathrm{mg} \mathrm{L}^{-1}$; por el contrario con oxicloruro de cobre se presentó un $100 \%$ de resistencia, empleándose la misma concentración. Los resultados obtenidos para sulfato de cobre concuerdan con los reportados por Quezado et al. (2003) y Mitrev y Kovacevic (2006), quienes obtuvieron un $100 \%$ de aislamientos sensibles de Xanthomonas sp. a este compuesto cúprico, empleando una concentración de $200 \mathrm{mg} \mathrm{L}^{-1}$.

Maneguim et al. (2007) también reportan sensibilidad de 122 aislamientos de $X$. axonopodis 
pv. citri a concentraciones de $100 \mathrm{mg} \mathrm{L}^{-1}$ de sulfato de cobre; pero al igual que Quezado et al. (2003) observaron resistencia a concentraciones de 1 a $50 \mathrm{mg} \mathrm{L}^{-1}$. Adicionalmente, para aislamientos de $P$. syringae se ha reportado sensibilidad a este compuesto a concentraciones de $300 \mathrm{mg} \mathrm{L}^{-1}$, pero a concentraciones de 40 $160 \mathrm{mg} \mathrm{L}^{-1}$ los aislamientos de esta especie no son sensibles (Spotts y Cervantes, 1995). Esto indica que para obtener un control adecuado de bacteriosis en distintas especies de plantas, es necesario realizar aplicaciones de sulfato de cobre superiores a $160 \mathrm{mg} \mathrm{L}^{-1}$, y para el caso de este estudio concentraciones de $750 \mathrm{mg} \mathrm{L}^{-1}$, ya que a altas concentraciones, los iones de cobre se unirían a grupos sulfhidrilo (-SH) de ciertos aminoácidos, causando la desnaturalización de proteínas y enzimas de las bacterias (Agrios, 2005). Por otra parte, los altos porcentajes de resistencia presentados para oxicloruro de cobre, pueden estar relacionados con las aplicaciones en las zonas productoras de gulupa en Colombia. Con anterioridad se ha reportado que el aislamiento de bacterias resistentes a productos a base de cobre, se encuentran relacionados con la aplicación constante de estos productos en diferentes especies de plantas (Maneguim et al., 2007), siendo la presión de selección de los bactericidas y antibióticos de acción específica la causa principal del desarrollo de resistencia. Además, este uso continuo de productos a base de cobre ha favorecido la movilización de elementos de resistencia entre las bacterias saprofitas y patógenas de las plantas (Quezado et al., 2003). Por otra parte, la mortalidad de diversas bacterias en contacto con cobre metálico se cree que está influenciado por la afluencia de iones de cobre en las células, pero el mecanismo no se entiende completamente (Elguindi et al., 2011); lo que revela que en el caso del oxicloruro de cobre, posiblemente la concentración de iones libres de cobre, puede ser menor que la obtenida en sulfato de cobre. Otra de las razones que podrían explicar la resistencia adquirida para el oxicloruro de cobre está relacionado con genes de resisten- cia (genes cop) que pueden estar localizados en plásmidos nativos, y que a concentraciones no inhibitorias de los compuestos cúpricos, pueden activarse.

\section{CONCLUSIONES}

Se desconocen otros trabajos similares a este, en el cual se analicen bacterias asociadas a bacteriosis en pasifloráceas en Colombia, en el presente se abarca una población significativa y representativa en número y zonas productoras de pasifloras en el país de pasifloráceas. El panorama planteado a partir de los resultados sugiere como alternativa el uso de sulfato de cobre para control bacteriano u otros metabolitos de origen microbiano que son generados de procesos de bioprospección para control de enfermedades de plantas, pero a partir de planeación y uso racional, como parte de estrategias de manejo de la resistencia al cobre, pues como se demuestra en el trabajo, debido a la presión selectiva que trae consigo el uso masivo de productos fitosanitarios concretamente productos cúpricos y antibióticos, se está en alto riesgo hacer inefectivo el control químico de las bacteriosis en gulupa. Es probable que la aparición de aislamientos poco sensibles a antibióticos en plantaciones de pasifloráceas, pueda estar relacionado, entre otros, con las prácticas culturales (rotación de cultivos no susceptibles a la bacteria, la eliminación de plantas arvenses y manejo de residuos de cosecha, entre otros) y la estabilidad de la resistencia a los agentes químicos (Quezado et al., 2003; Maneguim et al., 2007).

Los costos de aplicación de antibióticos en agricultura no han sido evaluados en el país en términos de la aparición de resistencia de microorganismos a estos, lo que conlleva a plantearse algunas preguntas como: żEl uso de antibióticos en la agricultura contribuye al desarrollo de resistencia a los antibióticos empleados para controlar patógenos humanos? Esta cuestión ha sido objeto de polémica debate en los últimos años, especial- 
mente en Europa y América del Norte. En la producción de alimentos, la censura se ha dirigido hacia el uso de antibióticos en la cría de animales (Khachatourians, 1998; Mcmanus et al., 2002). En casos como este, es necesario implementar acciones contra la aparición de cepas bacterianas resistentes a estos antimicrobiales y observar cuidadosamente los resultados de las aplicaciones en campo en la producción de frutas, para que esta pueda seguir siendo una actividad rentable.

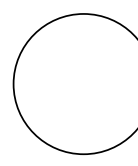

Agrios, G. 2005. Plant pathology. $5^{\text {th }}$ ed. Elsevier Academic Press, Amsterdam, The Netherlands.

Adaskaveg, J.E., G.F. Driever, H. Förster, W.D. Gubler, L.M. Wade y B. Holtz. 2008. Detection of Erwinia amylovora strains with reduced sensitivity to currently registered antibiotics and management of fire blight of pome fruits using kasugamycin in integrated strategies. Acta Hort. 793, 375-382.

Angulo, R. 2009. Gulupa. Passiflora edulis var. edulis Sims. Bayer CropScience, Bogotá.

Balaban, N.Q., J. Merrin, R. Chait, L. Kowalik y S. Leibler. 2004. Bacterial persistence as a phenotypic switch. Science 305, 1622-1625.

Behlau, F., J. Jones, M.E. Myers y J. Graham. 2012. Monitoring for resistant populations of Xanthomonas citri subsp. citri and epiphytic bacteria on citrus trees treated with copper or streptomycin using a new semi-selective medium. Eur. J. Plant Pathol. 132, 259-270.

Castillo, N. y G. Granada. 1995. Estudios sobre la bacteriosis en maracuyá en el Valle del Cauca: etiología, hospederos y control. Fitopat. Col. 19(1), 55-61.

Chinedum E. 2005. Microbial resistance to antibiotics. Afr. J. Biotechnol. 4(13), 1606-1611.

Cooksey, D. 1990. Genetics of bactericide resistance in plant pathogenic bacteria. Annu. Rev. Phytopathol. 28, 201-219.

Cooksey, D.A., H.R. Azad, J. Cha y C.K. Lim. 1990. Copper resistance gene homologs in pathogenic and saprophytic bacterial species from tomato. Appl. Environ. Microbiol. 56, 431-435.

\section{AGRADECIMIENTOS}

Este artículo fue resultado de un proyecto financiado por la Universidad Nacional de Colombia, Ministerio de Agricultura y Desarrollo Rural (contrato MADR 2007L4348-49) y Asociación Hortifrutícola de Colombia (proyecto Asohofrucol IB1350). Los autores expresan su agradecimiento a los señores Jesús León y Wadith de León por su ayuda técnica en laboratorio y a Marcela Jaramillo por la revisión del texto.

\section{REFERENCIAS BIBLIOGRÁFICAS}

Dámaso, D. 1990. Antibacterianos. Marketing Pharm S.A., Madrid.

Duffy, B., F. Walsh, C. Pelludat, E. Holliger, C. Oulevet y F. Widmer. 2011. Environmental monitoring of antibiotic resistance and impact of streptomycin use on orchard bacterial communities. Acta Hort. $896,483-488$

Elguindi, J., S. Moffitt, H. Hasman, C. Andrade, S. Raghavan y C. Rensing. C. 2011. Metallic copper corrosion rates, moisture content, and growth medium influence survival of copper ion-resistant bacteria. Appl. Microbiol. Biotechnol. 89, 1963 1970

Guerrero, E. y L. Hoyos-Carvajal. 2011. Buenas prácticas agrícolas (BPA) con énfasis en el manejo integrado de plagas y enfermedades de gulupa (Passiflora edulis Sims.). Produmedios, Bogotá.

Ishikawa, R., M. Suzuki-Nishimoto, A. Fukuchi y K. Matsuura. 2004. Effective control of cabbage black rot by validamycin A and its effect on extracellular polysaccharide-production of Xanthomonas campestris pv. campestris. J. Pest Sci. 29, 209-213.

Khachatourians, G. 1998. Agricultural use of antibiotics and the evolution and transfer of antibiotic-resistant bacteria. Can. Med. Assoc. J. 159(9), 119-1136

Llácer, G. 2000. Patología vegetal. 2a. ed. Tomo 1. Mundi-Prensa, Madrid.

Llop, P., S. Barbe y M. López. 2012. Functions and origin of plasmids in Erwinia species that are pathogenic to or epiphytically associated with pome fruit trees. Trees 26, 31-46 
Loper, J., M. Henkels, R. Roberts, M. Willett y T. Smith. 1991. Evaluation of streptomycin, oxytetracycline and copper resistance of Erwinia amylovora isolated from pear orchards in Washington State. Plant Dis. 75, 287-290.

McManus, P., V. Stockwell, G. Sundin y A. Jones. 2002. Antibiotic use in plant agriculture. Annu. Rev. Phytopathol. 40, 443-65

Maneguim, L., D. Rinaldi, A. Santos, L. Rodrigues, M. Silva, M. Canteri, M. y R. Leite. 2007. Sensibilidade de Xanthomonas axonopodis pv. citri ao cobre e mancozeb. Fitopatol. Bras. 32, 247-252.

Manicom, B., C. Ruggiero, R. Ploetz, R. y A. Goes. 2003. Disease of Passion Fruit. pp 413-442. En: Ploetz, R. (ed.). Diseases of tropical fruit crops. MA CABI Publishing, London.

Martin, H.L., V. Hamilton y R.A. Kopittke. 2004. Copper tolerance in Australian populations of Xanthomonas campestris pv. vesicatoria contributes to poor field control of bacterial spot of pepper. Plant Dis. 88, 921-924.

McGhee, G.C. y G. Sundin. 2011. Evaluation of kasugamycin for fire blight management, effect on non target bacteria, and assessment of kasugamycin resistance potential in Erwinia amylovora. Phytopathol. 101, 192-204.

Mitrev, S. y B. Kovacevic. 2006. Characterization of Xanthomonas axonopodis pv. vesicatoria isolated from peppers in Macedonia. J. Plant Parthol. 88(3), 321-324.

Oliveira, J., E. Silveira, R. Mariano, E. Cardoso e I. Viana, I. 2007. Caracterização de isolados de Acidovorax avenae subsp. citrulli. Fitopatol. Bras. 32, 480-487.

Pérez, D. 1998. Resistencia bacteriana a antimicrobianos: su importancia en la toma de decisiones en la práctica diaria. Información terapéutica del sistema nacional de salud 22(3), 58-67.
Pérez, L. 1994. Patología vegetal. Editorial León, Bogotá.

Quezado, A., A. Gazzoto, R. Leite y L. Camergo, L. 2003. Sensibilidade a cobre, estreptomicina e oxitetraciclina em Xanthomonas spp. associadas à mancha-bacteriana do tomate para processamento industrial. Hort. Brasil. 21(4), 670-675.

Russo, N., T. Bur, D. Breth y H. Aldwinckle. H. 2008. Isolation of streptomycin-resistant isolates of Erwinia amylovora in New York. Plant Dis. 92, 714-718.

Sales, R., I. Oliveira, R. Mariano, G. Silva, y G. Nunes. 2005. Efeito de kasugamicina e oxicloreto de cobre no controle da mancha aquosa do meloeiro. Fitopatol. Brasil. 30, 295-298.

Shenge, K., R. Mabagala y C. Mortensen. 2007. Identification and characterization of strains of Xanthomonas campestris pv. vesicatoria from Tanzania by biolog- system and sensitivity to antibiotics. Afr. J. Biotechnol. 6 (1), 15-22.

Spotts, R. y L. Cervantes. 1995. Copper, oxytetracycline, and streptomycin resistance of Pseudomonas syringae pv. syringae strains from pear orchards in Oregon and Washington. Plant Dis. 79(11), 1132 1135.

Thiele-Bruhn, S. 2003. Pharmaceutical antibiotic compounds in soils - a review. J. Plant Nutr. Soil Sci. $166,145-167$

Wiuff C., R. Zappala, R. Regoes, K. Garner, F. Baquero y B. Levin. 2005. Phenotypic tolerance: antibiotic enrichment of noninherited resistance in bacterial populations. Antimicrob. Agents Chemother 49(4), 1483-94.

Zhang, L., W.C. Chiang, O. Gao, M. Givskov, T. TolkerNielsen, L. Yang y G. Zhang. 2012. The catabolite repression control protein Crc plays a role in the development of antimicrobial-tolerant subpopulations in Pseudomonas aeruginosa biofilms. Microbiol. 158(12), 3014-3019. 